\title{
SUPERREPLICATION OF OPTIONS ON SEVERAL UNDERLYING ASSETS
}

\author{
ERIK EKSTRÖM, $* * *$ \\ SVANTE JANSON * AND \\ JOHAN TYSK, ${ }^{* * * *}$ Uppsala University
}

\begin{abstract}
We investigate the conditions on a hedger, who overestimates the (time- and leveldependent) volatility, to superreplicate a convex claim on several underlying assets. It is shown that the classic Black-Scholes model is the only model, within a large class, for which overestimation of the volatility yields the desired superreplication property. This is in contrast to the one-dimensional case, in which it is known that overestimation of the volatility with any time- and level-dependent model guarantees superreplication of convex claims.
\end{abstract}

Keywords: Parabolic equation; superreplication; convexity; option

2000 Mathematics Subject Classification: Primary 91B28

Secondary $60 \mathrm{H} 05 ; 35 \mathrm{~K} 10$

\section{Introduction}

For options written on one underlying asset, it is well known that convexity of the contract function ensures certain monotonicity properties of the option price with respect to the volatility. For example, if the contract function is convex, then the option price is an increasing function of the time- and level-dependent volatility - see Bergman et al. (1996), El Karoui et al. (1998), Hobson (1998), and Janson and Tysk (2003). It is also known that a hedger who overestimates the volatility will superreplicate a given convex claim - see El Karoui et al. (1998) and Hobson (1998). Crucial for both the monotonicity result and for the superreplication property, is the fact that the price of a convex claim is convex (as a function of the current stock price) at any time before maturity - see the references given above and also Martini (1995), (1999). This fact, however, applies in one-dimensional cases; it is easy to find examples of an option, with a convex payoff of two underlying assets, that has a nonconvex price - see Example 2.1 below, or Janson and Tysk (2003).

In the present article, we study convexity properties of prices of options on several underlying assets - also see Bergenthum and Rüschendorf (2004) - and show that the only convexitypreserving model, within a large class of standard models (to be defined below), is geometric Brownian motion (possibly with a time-dependent volatility). Consequently, if a hedger wants to use a standard model to superreplicate any convex claim by overestimation of the volatility in the sense of quadratic forms, then the only model he can use is geometric Brownian motion. Thus, there are rather few convexity-preserving models in several dimensions. It follows that one often needs to approximate the volatility, and therefore also the option price, rather crudely

Received 5 January 2004; revision received 12 August 2004.

* Postal address: Department of Mathematics, Uppsala University, Box 480, SE-751 06 Uppsala, Sweden.

** Email address: ekstrom@math.uu.se

*** Partially supported by the Swedish Research Council. 
when overestimating the true volatility. Another approach to the superreplication problem is via the so-called Black-Scholes-Barenblatt equation - see, for example, Avellaneda et al. (1995), Lyons (1995), Romagnoli and Vargiolu (2000), Vargiolu (2001), and Gozzi and Vargiolu (2002a), (2002b). This equation is a fully nonlinear parabolic equation, which, for convex onedimensional claims, reduces to the usual Black-Scholes equation, once again illustrating the fact that superhedging is a much simpler problem in one dimension than in several.

Janson and Tysk (2004) considered second-order parabolic differential equations of the form

$$
\frac{\partial G}{\partial t}=\mathcal{L} G
$$

for functions $G \equiv G(x, t)$, where the differential operator

$$
\mathcal{L}=\sum_{i, j=1}^{n} a_{i j}(\boldsymbol{x}, t) \frac{\partial^{2}}{\partial x_{i} \partial x_{j}}+\sum_{i=1}^{n} b_{i}(\boldsymbol{x}, t) \frac{\partial}{\partial x_{i}}+c(\boldsymbol{x}, t)
$$

is elliptic. The authors found a necessary and sufficient local condition on the operator $\mathcal{L}$ that guarantees that the unique solution to (1.1), satisfying appropriate growth conditions, remains convex at every time $t>0$, provided that the initial condition is convex. If $\mathcal{L}$ satisfies the condition it is said to be locally convexity preserving (LCP). In the present paper we use some of the results of Janson and Tysk (2004) to study the problem of convexity-preserving models in finance. The results of Janson and Tysk cannot be used directly in our setting, however. In particular, the LCP condition is not sufficient to ensure the convexity of option prices. As a consequence, we have not been able to prove our main result (Theorem 2.3, below) using arguments involving only PDE methods. Rather, we need to formulate the problem in terms of the diffusion matrix $\boldsymbol{\beta}$, and not in terms of the matrix $\boldsymbol{\beta} \boldsymbol{\beta}^{\top}$, which appears in the Black-Scholes equation.

The paper is organized as follows. In Section 2, we introduce the notion of convexitypreserving models and also the LCP condition. Then we describe the set of models under consideration, namely the so-called standard models, and present Theorem 2.3, which states that, for $n \geq 2$, the only standard, convexity-preserving model is geometric Brownian motion. We also provide a simple example in which convexity of the option price is lost. In Section 3, we show that if the hedger uses a convexity-preserving model, then overestimation of the diffusion matrix, in the sense of quadratic forms, guarantees superreplication of convex claims.

\section{Convexity-preserving models}

Consider a market consisting of a bank account with price process

$$
B(t)=B(0) \exp \left\{\int_{0}^{t} r(u) \mathrm{d} u\right\}
$$

where the interest rate $r$ is an integrable, deterministic function; and $n$ risky assets, with the price $X_{i}$ of the $i$ th asset satisfying the stochastic differential equation

$$
\mathrm{d} X_{i}=\mu_{i}(\boldsymbol{X}, t) \mathrm{d} t+\sum_{j=1}^{n} \beta_{i j}(\boldsymbol{X}, t) \mathrm{d} W_{j} .
$$

In this equation, the drift $\mu_{i}$ is some deterministic function of the current stock prices and time, $\boldsymbol{W}$ is an $n$-dimensional Brownian motion, and the diffusion matrix $\boldsymbol{\beta}=\left(\beta_{i j}(\boldsymbol{x}, t)\right)_{i, j=1}^{n}$ 
is assumed to be nonsingular for all $\boldsymbol{x}$ with positive components. Note that the only source of randomness in the diffusion matrix $\boldsymbol{\beta}$ is its dependence on the current stock prices. In finance, it is natural to consider stock prices that cannot become negative. Therefore we let 0 be an absorbing barrier, i.e. if $X_{i}$ is 0 at some time, then $X_{i}$ remains 0 forever.

We assume that the market is complete and arbitrage free. More precisely, given a finite time horizon $T>0$, we assume that there exists a unique probability measure $\tilde{\mathrm{P}}$, equivalent to the original measure $\mathrm{P}$, such that

$$
\mathrm{d} X_{i}=r(t) X_{i} \mathrm{~d} t+\sum_{j=1}^{n} \beta_{i j}(\boldsymbol{X}, t) \mathrm{d} \tilde{W}_{j}
$$

for some $\tilde{\mathrm{P}}$-Brownian motion $\tilde{\boldsymbol{W}}$, i.e. $B^{-1}(t) \boldsymbol{X}(t)$ is a local martingale up to time $T$ under $\tilde{\mathrm{P}}$. Conditions ensuring the existence and the uniqueness of the probability measure $\tilde{\mathrm{P}}$ can be found in, for example, Karatzas and Shreve (1998). Let $g: \overline{\mathbb{R}_{+}^{n}} \rightarrow \mathbb{R}$ be continuous and of at most polynomial growth, where $\overline{\mathbb{R}_{+}^{n}}=\left\{x \in \mathbb{R}^{n}: x_{i} \geq 0,1 \leq i \leq n\right\}$ and $\mathbb{R}_{+}^{n}$ is its interior. Standard arbitrage theory yields that at time $t$, the price of the option that at time $T_{0} \leq T$ pays $g\left(\boldsymbol{X}\left(T_{0}\right)\right)$ is $F(X(t), t)$, where

$$
F(\boldsymbol{x}, t)=\exp \left\{-\int_{t}^{T_{0}} r(u) \mathrm{d} u\right\} \tilde{\mathrm{E}}_{\boldsymbol{x}, t} g\left(\boldsymbol{X}\left(T_{0}\right)\right) .
$$

Here, $\tilde{\mathrm{E}}_{\boldsymbol{x}, t}$ denotes expected value with respect to the measure $\tilde{\mathrm{P}}$ and its indices indicate that $\boldsymbol{X}(t)=\boldsymbol{x}$. Moreover, this pricing function $F$ solves the Black-Scholes parabolic differential equation

where

$$
\frac{\partial F}{\partial t}+\mathcal{L} F=0
$$

$$
\mathcal{L}=\frac{1}{2} \sum_{i, j=1}^{n} a_{i j}(\boldsymbol{x}, t) \frac{\partial^{2}}{\partial x_{i} \partial x_{j}}+\sum_{i=1}^{n} r x_{i} \frac{\partial}{\partial x_{i}}-r,
$$

with terminal condition

$$
F\left(\boldsymbol{x}, T_{0}\right)=g(\boldsymbol{x}) .
$$

In this equation, the coefficients $a_{i j} \equiv a_{i j}(\boldsymbol{x}, t)$ are the entries of the $n \times n$ matrix $\boldsymbol{\beta} \boldsymbol{\beta}^{\top}$. Note that the invertibility of $\boldsymbol{\beta}$ guarantees the parabolicity of (2.3) (since the direction of the time variable is opposite to the customary one).

Assume that the diffusion coefficients $\beta_{i j}$ are linear in $x_{i}$ and independent of $x_{l}, l \neq i$. Then, under the measure $\tilde{\mathrm{P}}$, the stock-price vector $\boldsymbol{X}$ satisfies

$$
\mathrm{d} X_{i}=r(t) X_{i} \mathrm{~d} t+X_{i} \sum_{j=1}^{n} \sigma_{i j}(t) \mathrm{d} \tilde{W}_{j}
$$

for some deterministic functions $\sigma_{i j}(t)$. Such a process $\boldsymbol{X}$ is called $n$-dimensional geometric Brownian motion with time-dependent volatility or, simply, geometric Brownian motion. Thus, to show that a process $\boldsymbol{X}$ defined by (2.1) is geometric Brownian motion, one has to show that $\beta_{i j}(\boldsymbol{x}, t)=x_{i} \sigma_{i j}(t)$ for all $i$ and $j$.

We will say that a model for the stock-price vector $\boldsymbol{X}$ (or the diffusion matrix $\boldsymbol{\beta}$ ) is convexity preserving if, for any $T_{0} \leq T$, the price of an option with a convex payoff $g\left(\boldsymbol{X}\left(T_{0}\right)\right)$ at $T_{0}$ is convex in $\boldsymbol{X}(t)$ at all times $t$ prior to $T_{0}$. 
Theorem 2.1. Geometric Brownian motion is convexity preserving.

Proof. Let $T_{0} \leq T$. It is well known that if $\boldsymbol{X}$ is geometric Brownian motion as in (2.5), then

$$
X_{i}\left(T_{0}\right)=x_{i} \exp \left\{\int_{t}^{T_{0}}\left(r(u)-\frac{1}{2} \sum_{j=1}^{n} \sigma_{i j}^{2}(u)\right) \mathrm{d} u+\sum_{j=1}^{n} \int_{t}^{T_{0}} \sigma_{i j}(u) \mathrm{d} \tilde{W}_{j}\right\},
$$

where $x_{i}=X_{i}(t)$. Let $g: \mathbb{R}_{+}^{n} \rightarrow \mathbb{R}$ be convex. At time $t$, the price $F(\boldsymbol{X}(t), t)$ of an option that at time $T_{0}$ pays $g\left(\boldsymbol{X}\left(T_{0}\right)\right)$ is given by

$$
F(\boldsymbol{x}, t)=\exp \left\{-\int_{t}^{T_{0}} r(u) \mathrm{d} u\right\} \tilde{\mathrm{E}}_{\boldsymbol{x}, t} g\left(\boldsymbol{X}\left(T_{0}\right)\right),
$$

which is convex in $\boldsymbol{x}$ since $X_{i}\left(T_{0}\right)$ is linear in $x_{i}$ and $g$ is convex. Thus, geometric Brownian motion is convexity preserving.

Next, we investigate which other models are convexity preserving. It turns out that if one imposes some conditions on the diffusion matrix, then geometric Brownian motion is the only such model. We first introduce the LCP condition.

Definition 2.1. Assume that the coefficients of the differential operator $\mathcal{L}$ are in $C^{3}\left(\mathbb{R}_{+}^{n} \times\right.$ $[0, T])$. Let $\boldsymbol{x} \in \mathbb{R}_{+}^{n}$ and let $t \in[0, T]$. Then $\mathcal{L}$ is said to be LCP at $(\boldsymbol{x}, t)$ if

$$
D_{u u}(\mathcal{L} f)(\boldsymbol{x}, t) \geq 0
$$

whenever $\boldsymbol{u} \in \mathbb{R}^{n} \backslash\{\boldsymbol{0}\}, f \in C^{\infty}\left(\mathbb{R}_{+}^{n}\right)$ is convex in a neighborhood of $\boldsymbol{x}$, and $D_{\boldsymbol{u} \boldsymbol{u}} f(\boldsymbol{x})=0$. (Here, $D_{\boldsymbol{u} u}$ denotes differentiation twice in the $\boldsymbol{u}$-direction.)

If $G$ is a solution to (1.1) then the infinitesimal change of $G$ during a short time interval $\Delta t$ is approximately $\Delta t(\mathcal{L} G)$. Thus, the LCP condition is intuitively the correct condition to preserve convexity: if, at some instant, convexity is almost lost in some direction $\boldsymbol{u}$, then the infinitesimal change of $G$ is convex in that direction.

Henceforth, for simplicity, we will work under the following assumption.

Hypothesis 2.1. The diffusion matrix $\boldsymbol{\beta}$ is in $C\left(\overline{\mathbb{R}_{+}^{n}} \times[0, T]\right) \cap C^{3}\left(\mathbb{R}_{+}^{n} \times[0, T]\right)$ and is such that, for any vector of nonnegative initial values of the stocks, there exists a unique strong solution $\boldsymbol{X}$ to (2.1) with absorption at 0 of the ith component $X_{i}$, for all $i$.

We also assume that the diffusion matrix $\boldsymbol{\beta}$ is such that, for any smooth terminal value $g$, the function $F$ defined by (2.2) has continuous derivatives $D_{x}^{k} D_{t}^{m} F, m \in\{0,1\}, 0 \leq|k|+2 m \leq 4$, up to time $T_{0}$. Here, $D_{t}$ denotes the derivative with respect to time and $D_{x}^{k}$ denotes derivatives in the spatial variables as indicated by the multi-index $k$.

Remark 2.1. The assumption that $\boldsymbol{\beta}$ is $C^{3}$ is unnecessarily strong - see Section 3 of Janson and Tysk (2004). To clarify the presentation, however, we will continue to assume this.

Theorem 2.2. Let the diffusion matrix $\boldsymbol{\beta}$ satisfy Hypothesis 2.1, and let $\mathcal{L}$ be the corresponding differential operator, as in (2.4). If $\boldsymbol{\beta}$ is convexity preserving then $\mathcal{L}$ is LCP at all points $\left(x_{0}, T_{0}\right) \in \mathbb{R}_{+}^{n} \times(0, T)$.

Proof. Suppose that $f \in C^{\infty}\left(\mathbb{R}_{+}^{n}\right)$ is convex in a neighborhood of some point $\boldsymbol{x}_{0} \in \mathbb{R}_{+}^{n}$, and suppose that $D_{\boldsymbol{u} u} f\left(\boldsymbol{x}_{0}\right)=0$ for some direction $\boldsymbol{u} \neq 0$. Then, there exists a smooth convex function $g: \mathbb{R}_{+}^{n} \rightarrow \mathbb{R}$ that equals $f$ in a neighborhood of $\boldsymbol{x}_{0}$; for details, see Lemma 3.2 of 
Janson and Tysk (2004). Since $\boldsymbol{\beta}$ is convexity preserving, there exists a solution $F(\boldsymbol{x}, t)$ (defined by (2.2)) to (2.3), which is convex in $\boldsymbol{x}$ for all $t \leq T_{0}$, with terminal condition $F\left(\boldsymbol{x}, T_{0}\right)=g(\boldsymbol{x})$. By assumption, $D_{x}^{k} D_{t}^{m} F, m \in\{0,1\}, 0 \leq|k|+2 m \leq 4$, exist and are continuous up to time $T_{0}$. Since $D_{u \boldsymbol{u}} F\left(\boldsymbol{x}_{0}, t\right) \geq 0$ for all $t \leq T_{0}$ and $D_{\boldsymbol{u} u} F\left(\boldsymbol{x}_{0}, T_{0}\right)=0$, we have $D_{t} D_{u \boldsymbol{u}} F\left(\boldsymbol{x}_{0}, T_{0}\right) \leq 0$. Therefore, using (2.3),

$$
\begin{aligned}
D_{\boldsymbol{u и}}(\mathscr{L} f)\left(\boldsymbol{x}_{0}, T_{0}\right) & =D_{\boldsymbol{u u}}(\mathcal{L} F)\left(\boldsymbol{x}_{0}, T_{0}\right) \\
& =-D_{\boldsymbol{u} u}\left(D_{t} F\right)\left(x_{0}, T_{0}\right) \\
& =-D_{t} D_{u \boldsymbol{u}} F\left(\boldsymbol{x}_{0}, T_{0}\right) \\
& \geq 0 .
\end{aligned}
$$

Remark 2.2. Janson and Tysk (2004) showed that the LCP condition is both necessary and sufficient to guarantee that an operator $\mathcal{L}$ defined on $\mathbb{R}^{n} \times[0, T]$ is convexity preserving. To establish sufficiency in the present setting, we would have to add some conditions on the boundary of $\mathbb{R}_{+}^{n}$ in the definition of LCP. In our analysis, however, we only use local convexity preservation as a necessary condition, and we therefore side-step the rather technical considerations about the appropriate LCP condition for boundary points.

We now present the class of models under consideration.

Definition 2.2. A model with a diffusion matrix $\boldsymbol{\beta}$ that satisfies Hypothesis 2.1 is standard if the following conditions are satisfied:

(i) the diffusion coefficient $\beta_{i j}$ is a function only of $x_{i}$ and $t$;

(ii) the diffusion coefficients satisfy $\beta_{i j}=0$ for $x_{i}=0$; and

(iii) for all $i=1, \ldots, n$ and fixed times $t$, the volatility

$$
\sqrt{a_{i i}\left(x_{i}, t\right)} / x_{i}=\sqrt{\beta_{i 1}^{2}\left(x_{i}, t\right)+\cdots+\beta_{i n}^{2}\left(x_{i}, t\right)} / x_{i}
$$

of the $i$ th asset is not a monotonically increasing function of $x_{i}$, unless it is constant.

Remark 2.3. Note that condition (i) does not exclude dependence between the assets. Instead, it merely says that the volatility of the $i$ th asset depends only on the value of that asset and time. Note further that condition (ii) allows volatilities tending to infinity for asset values close to 0 . Condition (iii) seems to be satisfied for virtually all models of option pricing; in fact, many models have large volatility for small values of the underlying asset, and thus the volatility is strictly decreasing on some interval. However, without this condition, there are other convexity-preserving models - see Bergenthum and Rüschendorf (2004) and Janson and Tysk (2004).

We can now state our main theorem.

Theorem 2.3. Let $n \geq 2$. Then geometric Brownian motion (with time-dependent volatility) is the only standard model that is convexity preserving.

Remark 2.4. From Theorem 2.3 , it follows, for example, that the (standard) constant-elasticityof-variance (CEV) model, in which the price of the $i$ th asset is given under $\tilde{\mathrm{P}}$ by

$$
\mathrm{d} X_{i}=r(t) X_{i} \mathrm{~d} t+\sigma_{i} X_{i}^{\gamma_{i}} \mathrm{~d} \tilde{W}_{i}
$$

for some nonzero parameters $\sigma_{i}$ and $\gamma_{i}$, where $0<\gamma_{i}<1$, is not convexity preserving. 
In line with this remark, we give an explicit example of an option with a convex contract function but with a nonconvex price.

Example 2.1. Consider the interest-rate-free CEV model, in which the price of the $i$ th asset, $i=1,2$, is given under $\tilde{\mathrm{P}}$ by

$$
\mathrm{d} X_{i}=X_{i}^{\gamma} \mathrm{d} \tilde{W}_{i}
$$

where $0<\gamma<\frac{1}{2}$. Assume that the payoff is given by $g\left(X_{1}(T), X_{2}(T)\right)=\left(X_{1}(T)-X_{2}(T)\right)^{2}$ at time $T$. Then, the terminal time derivative of the option price satisfies

$$
\begin{aligned}
\frac{\partial F}{\partial t} & =-\frac{x_{1}^{2 \gamma}}{2} \frac{\partial^{2} F}{\partial x_{1}^{2}}-\frac{x_{2}^{2 \gamma}}{2} \frac{\partial^{2} F}{\partial x_{2}^{2}} \\
& =-x_{1}^{2 \gamma}-x_{2}^{2 \gamma} .
\end{aligned}
$$

Thus, the terminal time derivative is convex along the diagonal $x_{1}=x_{2}$, for example. Since $g=0$ along the diagonal, it follows that convexity is lost for times $t$ close to $T$.

Before proving Theorem 2.3, we need a technical lemma.

Lemma 2.1. Let $\boldsymbol{\beta}$ be a $C^{2}$ diffusion matrix such that $\beta_{i j}$ is a function of $x_{i}$ alone, and such that condition (ii) of Definition 2.2 is satisfied. Assume that there exist constants $B_{i j}$ such that

$$
\sum_{k=1}^{n} \beta_{i k}\left(x_{i}\right) \beta_{j k}\left(x_{j}\right)=B_{i j} x_{i} x_{j}
$$

for all $i$ and $j$ with $i \neq j$. Then there exists an index $l \in\{1, \ldots, n\}$ such that $\left(\beta_{l 1}, \ldots, \beta_{l n}\right)=$ $f\left(x_{l}\right) \boldsymbol{v}$ for some constant vector $\boldsymbol{v} \in \mathbb{R}^{n}$ and some function $f$.

If, in addition, (2.6) also holds for $i=j$, then $\boldsymbol{\beta}$ is the diffusion matrix of a geometric Brownian motion.

Proof. Let $\boldsymbol{\beta}_{i}=\left(\beta_{i 1}, \ldots, \beta_{i n}\right)$ and $\boldsymbol{\beta}_{i}^{\prime \prime}=\left(\beta_{i 1}^{\prime \prime}, \ldots, \beta_{i n}^{\prime \prime}\right)$ for $i=1, \ldots, n$. Here, the double primes refer to differentiation with respect to the $x_{i}$-variables. If $\boldsymbol{\beta}_{i}^{\prime \prime} \equiv 0$ for some $i$, then all of the components of $\boldsymbol{\beta}_{i}$ are affine functions of $x_{i}$. From Definition 2.2(ii), it follows that the components are, in fact, linear and, thus, the lemma is true in this particular case.

Now assume that we can choose coordinates $x_{1}, \ldots, x_{n}>0$ such that $\boldsymbol{\beta}_{i}^{\prime \prime}\left(x_{i}\right) \neq 0$. From (2.6) we have

$$
\boldsymbol{\beta}_{i}^{\prime \prime}\left(x_{i}\right) \cdot \boldsymbol{\beta}_{j}\left(x_{j}\right)=0
$$

for $i \neq j$. Since $\boldsymbol{\beta}_{1}\left(x_{1}\right), \ldots, \boldsymbol{\beta}_{n}\left(x_{n}\right)$ are linearly independent (recall that the diffusion matrix is assumed to be nonsingular), it follows from (2.7) that $\boldsymbol{\beta}_{1}^{\prime \prime}\left(x_{1}\right), \ldots, \boldsymbol{\beta}_{n}^{\prime \prime}\left(x_{n}\right)$ are linearly independent. Now, keeping $x_{1}, \ldots, x_{n-1}$ fixed, it follows from (2.7) that, for any $x_{n}, \boldsymbol{\beta}_{n}\left(x_{n}\right)$ is in the orthogonal (one-dimensional) complement of $\boldsymbol{\beta}_{1}^{\prime \prime}\left(x_{1}\right), \ldots, \boldsymbol{\beta}_{n-1}^{\prime \prime}\left(x_{n-1}\right)$. Thus, $\boldsymbol{\beta}_{n}\left(x_{n}\right)=$ $f\left(x_{n}\right) \boldsymbol{v}$ for some constant vector $\boldsymbol{v} \in \mathbb{R}^{n}$, which finishes the first part of the lemma.

Next, assume that (2.6) also holds for $i=j$, i.e. that $a_{i i}\left(x_{i}\right)=B_{i i} x_{i}^{2}$ for some positive constants $B_{i i}$. Fix $x_{2}, \ldots, x_{n}$ and let $\boldsymbol{v}_{i}=\boldsymbol{\beta}_{i}\left(x_{i}\right) / x_{i}$ for $i=2, \ldots, n$. Then

$$
\frac{\boldsymbol{\beta}_{1}\left(x_{1}\right)}{x_{1}} \cdot \boldsymbol{v}_{i}=B_{1 i}
$$

for all $x_{1}$. Since $\boldsymbol{v}_{i}, i=2, \ldots, n$, are linearly independent, it follows that

$$
\frac{\boldsymbol{\beta}_{1}\left(x_{1}\right)}{x_{1}} \in\left\{\boldsymbol{\beta}_{1}(1)+t \boldsymbol{w}, t \in \mathbb{R}\right\}
$$


for some vector $\boldsymbol{w} \in \mathbb{R}^{n}$. However, since the inner product

$$
\frac{\boldsymbol{\beta}_{1}\left(x_{1}\right)}{x_{1}} \cdot \frac{\boldsymbol{\beta}_{1}\left(x_{1}\right)}{x_{1}}=B_{11}
$$

is constant, we find that $\boldsymbol{\beta}_{1}\left(x_{1}\right) / x_{1}$ is a vector of constant length. By continuity, it follows that $\boldsymbol{\beta}_{1}\left(x_{1}\right) / x_{1}$ is a constant vector. Similarly, we deduce that all entries in the $i$ th row of $\boldsymbol{\beta}$ are linear in $x_{i}$ and, thus, the lemma follows.

Proof of Theorem 2.3. Assume that $\boldsymbol{\beta}$ is a standard diffusion matrix, which is convexity preserving, and let $\mathcal{L}$ be the corresponding differential operator appearing in the Black-Scholes equation (2.3). We suppress the time variable $t$ in the calculations below. First, choose two components $x_{i}$ and $x_{j}$. For a fixed $s$, let $f\left(x_{i}, x_{j}\right)=\frac{1}{2}\left(s x_{i}-x_{j}\right)^{2}$. Then $f$ is constant along the lines $x_{j}=s x_{i}+x_{i, 0}$, where $x_{i, 0}$ is a constant. If $\boldsymbol{u}=\boldsymbol{e}_{i}+s \boldsymbol{e}_{j}$ (where $\boldsymbol{e}_{i}$ and $\boldsymbol{e}_{j}$ are unit vectors in the $i$ - and $j$-directions, respectively) then, since $\mathcal{L}$ is LCP by Theorem 2.2, it follows that

$$
\begin{aligned}
0 & \leq 2 D_{\boldsymbol{u} \boldsymbol{u}}(\mathcal{L} f) \\
& =\left(\partial_{x_{i}}^{2}+2 s \partial_{x_{i}} \partial_{x_{j}}+s^{2} \partial_{x_{j}}^{2}\right)\left(\sum_{i, j=1}^{n} a_{i j} \frac{\partial^{2} f}{\partial x_{i} \partial x_{j}}+2 r \sum_{i=1}^{n} x_{i} \frac{\partial f}{\partial x_{i}}-2 r f\right) \\
& =\left(\partial_{x_{i}}^{2}+2 s \partial_{x_{i}} \partial_{x_{j}}+s^{2} \partial_{x_{j}}^{2}\right)\left(s^{2} a_{i i}-2 s a_{i j}+a_{j j}+2 r x_{i} f_{x_{i}}+2 r x_{j} f_{x_{j}}-2 r f\right) \\
& =-2 s^{3} \partial_{x_{j}}^{2} a_{i j}+s^{2}\left(\partial_{x_{i}}^{2} a_{i i}-4 \partial_{x_{i}} \partial_{x_{j}} a_{i j}+\partial_{x_{j}}^{2} a_{j j}\right)-2 s \partial_{x_{i}}^{2} a_{i j} .
\end{aligned}
$$

Since $s$ is arbitrary, we find that

$$
\partial_{x_{j}}^{2} a_{i j}=\partial_{x_{i}}^{2} a_{i j}=0
$$

and, so, $a_{i j}=B_{i j} x_{i} x_{j}+B_{1} x_{i}+B_{2} x_{j}+B_{3}$. The condition (ii) guarantees that $a_{i j}\left(x_{i}, x_{j}\right)$ vanishes for $x_{i}=0$ and for $x_{j}=0$, from which it follows that $B_{1}=B_{2}=B_{3}=0$. Hence, $a_{i j}=B_{i j} x_{i} x_{j}$ for $i \neq j$.

It remains to show that the same holds for the diagonal elements $a_{i i}$. Using the first part of Lemma 2.1, we may assume that, say, $\boldsymbol{\beta}_{n}\left(x_{n}\right)=f\left(x_{n}\right) \boldsymbol{v}$ for some constant vector $\boldsymbol{v} \in \mathbb{R}^{n}$. Now choose a row $\boldsymbol{\beta}_{i}, i \in\{1, \ldots, n-1\}$, in the diffusion matrix and consider the $2 \times 2$ matrix block

$$
\boldsymbol{A}_{i n}=\left(\begin{array}{cc}
a_{i i} & a_{i n} \\
a_{n i} & a_{n n}
\end{array}\right)=\left(\begin{array}{cc}
a_{i i}\left(x_{i}\right) & B_{i n} x_{i} x_{n} \\
B_{i n} x_{i} x_{n} & a_{n n}\left(x_{n}\right)
\end{array}\right) .
$$

Since $\mathcal{L}$ is LCP, the operator corresponding to $\boldsymbol{A}_{\text {in }}$ must be LCP for equations in the spatial variables $x_{i}$ and $x_{n}$. There are two different cases to consider. First, assume that $B_{i n}=0$. Then

$$
\boldsymbol{A}_{i n}=\left(\begin{array}{cc}
g^{2}\left(x_{i}\right) & 0 \\
0 & h^{2}\left(x_{n}\right)
\end{array}\right),
$$

where $g$ and $h$ are some positive functions. From Example 5.3 of Janson and Tysk (2004), we see that, in order to have a convexity-preserving operator, we must have

$$
g\left(x_{i}\right) g^{\prime \prime}\left(x_{i}\right)+h\left(x_{n}\right) h^{\prime \prime}\left(x_{n}\right) \geq 0
$$

for all $x_{i}$ and $x_{n}$. Hence, at least one of $g$ and $h$ is convex. Let this function be $g$. Then, by Definition 2.2(ii), we conclude that $g(0)=0$ and, from condition (iii), the function $g$ is seen 
to be linear, using the fact that $g\left(x_{i}\right) / x_{i}=\left[a_{i i}\left(x_{i}, t\right)\right]^{1 / 2} / x_{i}$ is increasing, since $g$ is a convex function vanishing at 0 . Therefore, the above inequality has only one term, so $h$ is also convex and linear, by the same argument. Hence, $a_{i i}\left(x_{i}\right)=C_{1}^{2} x_{i}^{2}$ and $a_{n n}\left(x_{n}\right)=C_{2}^{2} x_{n}^{2}$ for some constants $C_{1}$ and $C_{2}$.

Next, if $B_{\text {in }} \neq 0$ then

$$
a_{i n}\left(x_{i}, x_{n}\right)=B_{i n} x_{i} x_{n}=\boldsymbol{\beta}_{i}\left(x_{i}\right) \cdot \boldsymbol{\beta}_{n}\left(x_{n}\right)=f\left(x_{n}\right)\left(\boldsymbol{\beta}_{i}\left(x_{i}\right) \cdot \boldsymbol{v}\right)
$$

for some constant vector $\boldsymbol{v}$. It follows that $\boldsymbol{\beta}_{n}\left(x_{n}\right)=x_{n} \boldsymbol{u}$ for some constant vector $\boldsymbol{u} \in \mathbb{R}^{n}$. Therefore,

$$
\boldsymbol{A}_{\text {in }}=\left(\begin{array}{cc}
g^{2}\left(x_{i}\right)+D^{2} x_{i}^{2} & D E x_{i} x_{n} \\
D E x_{i} x_{n} & E^{2} x_{n}^{2}
\end{array}\right)
$$

for some nonzero constants $D$ and $E$ and some function $g$, which is strictly positive for $x_{i}>0$. From Corollary 5.2 of Janson and Tysk (2004), it follows that

$$
D_{\text {uu }} \sqrt{b^{2} a_{i i}\left(x_{i}\right)-2 a b D E x_{i} x_{n}+a^{2} E^{2} x_{n}^{2}} \geq 0
$$

for all directions $\boldsymbol{u}=a \boldsymbol{e}_{i}+b \boldsymbol{e}_{n}$. Direct calculations show that

$$
\begin{aligned}
D_{\boldsymbol{u u}} \sqrt{b^{2} a_{i i}\left(x_{i}\right)-2 a b D E x_{i} x_{n}+a^{2} E^{2} x_{n}^{2}} \\
=a^{2} b^{2}\left(b^{2} a_{i i}\left(x_{i}\right)-2 a b D E x_{i} x_{n}+a^{2} E^{2} x_{n}^{2}\right)^{-3 / 2} \\
\quad \times\left(\left(b(D-E) g\left(x_{i}\right)-\left(b D x_{i}-a E x_{n}\right) g^{\prime}\left(x_{i}\right)\right)^{2}\right. \\
\left.\quad+\left(b^{2} g^{2}\left(x_{i}\right)+\left(b D x_{i}-a E x_{n}\right)^{2}\right) g\left(x_{i}\right) g^{\prime \prime}\left(x_{i}\right)\right) .
\end{aligned}
$$

Here,

$$
b^{2} a_{i i}-2 a b D E x_{i} x_{n}+a^{2} E^{2} x_{n}^{2}=b^{2} g^{2}\left(x_{i}\right)+\left(b D x_{i}-a E x_{n}\right)^{2}>0
$$

if $b \neq 0$ and $x_{i}>0$. We claim that

$$
g^{\prime \prime}\left(x_{i}\right) \geq 0
$$

for all $x_{i}$. Indeed, for a given $x_{i}$ and $x_{n}$, let $K_{1}=E x_{n} g^{\prime}\left(x_{i}\right)$ and $K_{2}=(D-E) g\left(x_{i}\right)-$ $D x_{i} g^{\prime}\left(x_{i}\right)$. Then we know that

$$
\left(K_{1} a+K_{2} b\right)^{2}+\left(b^{2} g^{2}\left(x_{i}\right)+\left(b D x_{i}-a E x_{n}\right)^{2}\right) g\left(x_{i}\right) g^{\prime \prime}\left(x_{i}\right) \geq 0
$$

for all $a, b \neq 0$. If $K_{1}$ and $K_{2}$ both are 0 , then (2.8) follows immediately, while, if $K_{1}$ and $K_{2}$ are both nonzero, then $a, b \neq 0$ can be chosen such that $K_{1} a+K_{2} b=0$ and, thus, (2.8) follows. If $K_{1}=0, K_{2} \neq 0$, and $g\left(x_{i}\right) g^{\prime \prime}\left(x_{i}\right)<0$, then having $b=1$ and $a$ very large yields a contradiction, from which (2.8) follows - the case $K_{1} \neq 0=K_{2}$ is similar. Considering the inequality (2.8) together with conditions (ii) and (iii) of Definition 2.2, we find that $a_{i i}\left(x_{i}\right)=C^{2} x_{i}^{2}$ for some constant $C$. 
Remark 2.5. Theorem 2.3 tells us that, in order to ensure the preservation of convexity, regardless of the (convex) contract function, geometric Brownian motion should be used to model the asset prices. However, given a particular convex claim, it can of course be the case that other models also guarantee convex option prices. For example, if the contract function is $g\left(x_{1}, x_{2}\right)=g_{1}\left(x_{1}\right)+g_{2}\left(x_{2}\right)$, where $g_{1}$ and $g_{2}$ are both convex, then all models with diagonal diffusion matrices satisfying condition (i) of Definition 2.2 are convexity preserving. The reason for this is that such a claim is the sum of two one-dimensional claims, both of which have convex prices.

\section{Superreplication of convex claims}

Assume that an option-writer believes that the diffusion matrix $\boldsymbol{\beta}$ is as in (2.1), whereas the true stock-price vector $\tilde{\boldsymbol{X}}$ evolves according to

$$
\mathrm{d} \tilde{X}_{i}=\tilde{\mu}_{i}(\tilde{\boldsymbol{X}}, t) \mathrm{d} t+\sum_{j=1}^{n} \tilde{\beta}_{i j}(\tilde{\boldsymbol{X}}, t) \mathrm{d} W_{j}
$$

for some functions $\tilde{\mu}_{i}$ and $\tilde{\beta}_{i j}$. He will then (incorrectly) price an option on the stocks according to (2.2), where $\boldsymbol{X}$ is a diffusion with diffusion matrix $\boldsymbol{\beta}$. Moreover, if he tries to replicate the option with the hedging strategy suggested by his model, he will form a self-financing portfolio that has initial value $F(\boldsymbol{X}(0), 0)$ and is such that, at each instant $t$, it contains $\partial F(\tilde{\boldsymbol{X}}(t), t) / \partial x_{i}$ shares of the $i$ th asset (with the remaining amount invested in the bank account). In this section, we provide conditions under which the terminal value of the hedger's portfolio exceeds the option payoff $g\left(\boldsymbol{X}\left(T_{0}\right)\right)$ almost surely.

Given two diffusion matrices $\boldsymbol{\beta}$ and $\tilde{\boldsymbol{\beta}}$, we say that $\boldsymbol{\beta}$ dominates $\tilde{\boldsymbol{\beta}}$ if

$$
\boldsymbol{A}(\boldsymbol{x}, t)=\boldsymbol{\beta}(\boldsymbol{x}, t) \boldsymbol{\beta}^{\top}(\boldsymbol{x}, t) \geq \tilde{\boldsymbol{\beta}}(\boldsymbol{x}, t) \tilde{\boldsymbol{\beta}}^{\top}(\boldsymbol{x}, t)=\tilde{\boldsymbol{A}}(\boldsymbol{x}, t),
$$

as quadratic forms, for all $\boldsymbol{x}$ and $t$.

Theorem 3.1. Assume that a hedger overestimates the volatility, i.e. he uses a diffusion matrix $\boldsymbol{\beta}$ that dominates the true diffusion matrix $\tilde{\boldsymbol{\beta}}$. Moreover, assume that $\boldsymbol{\beta}$ is convexity preserving. Then, the hedger will superreplicate any convex claim written on $\boldsymbol{X}$. A hedger who underestimates the volatility, i.e. uses a $\boldsymbol{\beta}$ dominated by $\tilde{\boldsymbol{\beta}}$, will, in the same circumstances, subreplicate such a claim.

Theorem 3.1 is proved along the same lines as in the one-dimensional case-see, for example, El Karoui et al. (1998). For the reader's convenience, we give the proof here.

Proof. Let $g: \mathbb{R}_{+}^{n} \rightarrow \mathbb{R}$ be a convex contract function, and define $F$ as in (2.2). Since $\boldsymbol{\beta}$ is convexity preserving, $F(\boldsymbol{x}, t)$ is convex in $\boldsymbol{x}$ for all $t \in\left[0, T_{0}\right]$. The value $V(t)$ of the self-financing portfolio with $\partial F(\tilde{\boldsymbol{X}}(t), t) / \partial x_{i}$ shares of the $i$ th asset and initial value $V(0)=$ $F(\tilde{\boldsymbol{X}}(0), 0)$ has the dynamics

$$
\mathrm{d} V=r\left(V(t)-\sum_{i=1}^{n} \tilde{X}_{i}(t) \frac{\partial F}{\partial x_{i}}(\tilde{\boldsymbol{X}}(t), t)\right) \mathrm{d} t+\sum_{i=1}^{n} \frac{\partial F}{\partial x_{i}}(\tilde{\boldsymbol{X}}(t), t) \mathrm{d} \tilde{X}_{i}(t)
$$


Consider the process $Y(t):=V(t)-F(\tilde{\boldsymbol{X}}(t), t)$. From Itô's formula, it follows that

$$
\begin{aligned}
\mathrm{d} Y= & \mathrm{d} V-\frac{\partial F}{\partial t}(\tilde{\boldsymbol{X}}(t), t) \mathrm{d} t-\sum_{i=1}^{n} \frac{\partial F}{\partial x_{i}}(\tilde{\boldsymbol{X}}(t), t) \mathrm{d} \tilde{X}_{i} \\
& -\frac{1}{2} \sum_{i=1}^{n} \sum_{j=1}^{n} \tilde{a}_{i j}(\tilde{\boldsymbol{X}}(t), t) \frac{\partial^{2} F}{\partial x_{i} \partial x_{j}}(\tilde{\boldsymbol{X}}(t), t) \mathrm{d} t \\
= & r V(t) \mathrm{d} t-\frac{\partial F}{\partial t}(\tilde{\boldsymbol{X}}(t), t) \mathrm{d} t-r \sum_{i=1}^{n} \tilde{X}_{i}(t) \frac{\partial F}{\partial x_{i}}(\tilde{\boldsymbol{X}}(t), t) \mathrm{d} t \\
& -\frac{1}{2} \sum_{i=1}^{n} \sum_{j=1}^{n} \tilde{a}_{i j}(\tilde{\boldsymbol{X}}(t), t) \frac{\partial^{2} F}{\partial x_{i} \partial x_{j}}(\tilde{\boldsymbol{X}}(t), t) \mathrm{d} t \\
= & r(V(t)-F(\tilde{\boldsymbol{X}}(t), t)) \mathrm{d} t \\
& +\frac{1}{2} \sum_{i=1}^{n} \sum_{j=1}^{n}\left(a_{i j}(\tilde{\boldsymbol{X}}(t), t)-\tilde{a}_{i j}(\tilde{\boldsymbol{X}}(t), t)\right) \frac{\partial^{2} F}{\partial x_{i} \partial x_{j}}(\tilde{\boldsymbol{X}}(t), t) \mathrm{d} t \\
= & r Y(t) \mathrm{d} t+\frac{1}{2} \sum_{i=1}^{n} \sum_{j=1}^{n}\left(a_{i j}(\tilde{\boldsymbol{X}}(t), t)-\tilde{a}_{i j}(\tilde{\boldsymbol{X}}(t), t)\right) \frac{\partial^{2} F}{\partial x_{i} \partial x_{j}}(\tilde{\boldsymbol{X}}(t), t) \mathrm{d} t,
\end{aligned}
$$

where we have used the fact that $F$ solves the Black-Scholes equation (2.3) with diffusion matrix $\boldsymbol{\beta}$. Now, assume that the hedger overestimates the volatility, i.e. that $\boldsymbol{\beta}(\boldsymbol{x}, t)$ dominates $\tilde{\boldsymbol{\beta}}(\boldsymbol{x}, t)$ for all $\boldsymbol{x}$ and $t$. Then, since $F$ is convex, the last double sum is nonnegative. Thus, $Y(0)=0$ and $Y\left(T_{0}\right) \geq 0$. It follows that the final value $V\left(T_{0}\right)$ of the hedger's portfolio satisfies $V\left(T_{0}\right) \geq F\left(\tilde{\boldsymbol{X}}\left(T_{0}\right), \bar{T}_{0}\right)=g\left(\tilde{\boldsymbol{X}}\left(T_{0}\right)\right)$, meaning that the hedger superreplicates. The case of underestimation of the volatility is similar.

Theorems 2.3 and 3.1 show that if a hedger wants to be sure to superreplicate a convex claim on several underlying assets, then he should overestimate the true diffusion matrix $\tilde{\boldsymbol{\beta}}$ with a diffusion matrix $\boldsymbol{\beta}$ such that $\beta_{i j}(\boldsymbol{x}, t)=x_{i} \sigma_{i j}(t)$ for some functions $\sigma_{i j}(t)$. Note that there is no assumption that the true diffusion matrix be LCP; what matters is that the hedger should use a model that is LCP. Actually, it is not even essential that the true diffusion matrix $\tilde{\boldsymbol{\beta}}$ be a function of time and the current stock prices: the theorem is also true in the case that $\tilde{\boldsymbol{\beta}}$ is some adapted process dominated by $\boldsymbol{\beta}(\tilde{\boldsymbol{X}}(t), t)$ for all $t$, almost surely. This is, of course, in analogy with the one-dimensional case - see El Karoui et al. (1998).

Remark 3.1. By considering an American option as the limit of a sequence of European options (for the one-dimensional case, see El Karoui et al. (1998) or Ekström (2004)) it is clear that a model that gives convex European option prices also gives convex American option prices. For American claims, an option-writer must be sure that the value $V$ of his hedging portfolio at each instant $t$ satisfies $V(t) \geq g(\tilde{\boldsymbol{X}}(t))$. It can be shown that this is indeed the case if the option-writer overestimates the diffusion matrix with a convexity-preserving model.

Remark 3.2. Janson and Tysk (2004) showed that if $\boldsymbol{A} \geq \tilde{\boldsymbol{A}}$, as quadratic forms, and if either $\boldsymbol{A}$ or $\tilde{\boldsymbol{A}}$ is convexity preserving, then the corresponding solutions $F$ and $\tilde{F}$ satisfy the inequality $F(\boldsymbol{x}, t) \geq \tilde{F}(\boldsymbol{x}, t)$ for all $\boldsymbol{x}$ and $t$. Thus, if one knows that the true diffusion matrix can be bounded both above and below by some diffusion matrices corresponding to geometric 
Brownian motions, then one also has upper and lower bounds for the price of an option. This can also be seen as a consequence of Theorem 3.1.

It is also possible to formulate a converse to Theorem 3.1: if the hedger overestimates the volatility but uses a model that is not convexity preserving, then he cannot be sure to superreplicate. Rather than formulating this as a general theorem, we end this article with an example showing that preservation of convexity is indeed essential for the superreplication property to hold.

Example 3.1. Assume that $r=0$ and that a diffusion matrix $\boldsymbol{\beta}$ and a convex payoff function are specified so that the second derivative $D_{u u} F$ of the option price $F$ is strictly negative in some direction $\boldsymbol{u} \in \mathbb{R}^{n} \backslash\{\boldsymbol{0}\}$ at some point $\left(\boldsymbol{x}_{0}, t_{0}\right)$. Consider the two diffusion matrices $\boldsymbol{\beta}_{1}$ and $\boldsymbol{\beta}_{2}$ defined by

$$
\boldsymbol{\beta}_{1}(\boldsymbol{x}, t)= \begin{cases}\boldsymbol{\beta}(\boldsymbol{x}, t) & \text { if } t_{0}<t \leq T \\ \mathbf{0} & \text { if } t \leq t_{0}\end{cases}
$$

and

$$
\boldsymbol{\beta}_{2}(\boldsymbol{x}, t)= \begin{cases}\boldsymbol{\beta}(\boldsymbol{x}, t) & \text { if } t_{0}<t \leq T \\ \boldsymbol{\alpha} & \text { if } t \leq t_{0}\end{cases}
$$

where $\boldsymbol{\alpha}$ is some constant matrix such that

$$
\sum_{i, j} a_{i j} \frac{\partial^{2} F}{\partial x_{i} \partial x_{j}}<0
$$

with $\left(a_{i j}\right)=\boldsymbol{\alpha} \boldsymbol{\alpha}^{\top}$. It follows that $\boldsymbol{\beta}_{2}$ dominates $\boldsymbol{\beta}_{1}$. Moreover, if $F^{(i)}$ is the option price when the diffusion matrix is given by $\boldsymbol{\beta}_{i}$, then

$$
\frac{\partial F^{(2)}}{\partial t}=-\sum_{i, j} a_{i j} \frac{\partial^{2} F}{\partial x_{i} \partial x_{j}}>0=\frac{\partial F^{(1)}}{\partial t}
$$

at the point $\left(\boldsymbol{x}_{0}, t_{0}\right)$. Consequently, the price $F^{(2)}$ is smaller than $F^{(1)}$ at some times prior to $t_{0}$. Thus the superreplication property cannot hold if convexity is being lost.

Alternatively, to avoid degenerate diffusion matrices, one might add $\alpha$ to the diffusion matrix $\boldsymbol{\beta}$ for all times prior to $t_{0}$, to arrive at a similar result.

\section{References}

Avellaneda, M., Levy, A. And Parás, A. (1995). Pricing and hedging derivative securities in markets with uncertain volatilities. Appl. Math. Finance 2, 73-88.

Bergenthum, J. AND RÜsCHENDORF, L. (2004). Comparison of option prices in semimartingale models. Preprint, Freiburg University.

Bergman, Y.Z., Grundy, D. B. And Wiener, Z. (1996). General properties of option prices. J. Finance 51, 1573-1610.

Ekström, E. (2004). Properties of American option prices. Stoch. Process. Appl. 114, 265-278.

El Karoui, N., Jeanblanc-Picqué, M. And Shreve, S. (1998). Robustness of the Black and Scholes formula. Math. Finance 8, 93-126.

GozZI, F. AND VARGIOLU, T. (2002a). Superreplication of European multiasset derivatives with bounded stochastic volatility. Math. Meth. Operat. Res. 55, 69-91.

GozZI, F. AND VARGiolu, T. (2002b). On the superreplication approach for European interest rate derivatives. In Seminar on Stochastic Analysis, Random Fields and Applications, III (Ascona, 1999; Progress Prob. 52), eds R. C. Dalang and F. Russo, Birkhäuser, Basel, pp. 173-188.

Hobson, D. (1998). Volatility misspecification, option pricing and superreplication via coupling. Ann. Appl. Prob. 8, 193-205. 
Janson, S. And Tysk, J. (2003). Volatility time and properties of option prices. Ann. Appl. Prob. 13, 890-913.

JANSON, S. AND Tysk, J. (2004). Preservation of convexity of solutions to parabolic equations. J. Differential Equat. 206, 182-226.

Karatzas, I. And Shreve, S. E. (1998). Methods of Mathematical Finance (Appl. Math. 39). Springer, New York.

LyONS, T. J. (1995). Uncertain volatility and the risk-free synthesis of derivatives. Appl. Math. Finance 2, 117-133.

Martini, M. (1995). Propagation de la convexité par des semi-groupes martingaliens sur la demi-droite. Doctoral Thesis, Evry University.

Martini, M. (1999). Propagation of convexity by Markovian and martingalian semigroups. Potential Anal. 10, 133-175.

Romagnoli, S. And Vargiolu, T. (2000). Robustness of the Black-Scholes approach in the case of options on several assets. Finance Stoch. 4, 325-341.

VARgiolu, T. (2001). Existence, uniqueness and smoothness for the Black-Scholes-Barenblatt equation. Tech. Rep., Department of Pure and Applied Mathematics, University of Padova. 